\title{
Statistical facilitation and the redundant signals effect: What are race and coactivation models?
}

\author{
Jeff Miller ${ }^{1}$
}

Published online: 10 November 2015

(C) The Psychonomic Society, Inc. 2015

\begin{abstract}
As a supplement to Gondan and Minakata's (2015) tutorial on methods for testing the race model inequality, this theoretical note attempts to clarify further (a) the types of models that obey and violate the inequality and (b) the conclusions that can be drawn when the inequality is violated. In particular, the idea that individual racers proceed at the same speed in the single and redundant conditions (also known as "context independence") is shown to be better understood as an inherent part of Raab's (1962) original race model than as a separate, additional assumption. Thus, evidence that individual racers proceeded at different speeds in the single and redundant conditions, if available, should be viewed as supporting one type of coactivation model rather than an alternative model. In addition, it is shown that a class of racelike models without the assumption of context independence is so broad that it can never be falsified.
\end{abstract}

Keywords Race models $\cdot$ Race model inequality $\cdot$ Context independence $\cdot$ Coactivation

As is discussed by Gondan and Minakata (2015), separate activation or "race" models have been considered as possible explanations of redundant signals effects (RSEs) within a wide variety of research domains. There seem to be at least two reasons for this. First, the metaphor of a race is simple to understand and - in many domains - quite plausible. Second, the race model inequality (RMI; e.g., Miller, 1982) allows race

Jeff Miller

miller@psy.otago.ac.nz

1 Department of Psychology, University of Otago, PO Box 56, Dunedin 9054, New Zealand models to be tested, given properly arranged experimental conditions and appropriate data analyses (Gondan \& Minakata, 2015). When experimental results violate the RMI, researchers have generally concluded that the results support the alternative class of "coactivation" models. In general, these models "assume integrated processing of the combined stimuli" (Gondan \& Minakata, 2015), and they "allow both components of a redundant signal to influence responding on a single trial" (Miller, 1982, p. 248). Specific coactivation models often assume that activation is summed across channels to satisfy a response criterion (e.g., Miller, 1982; Miller \& Ulrich, 2003; Schwarz, 1989, 1994), although other kinds of coactivation models can also violate the RMI.

The comparison of race and coactivation models using the RMI has been complicated by suggestions that some race models also allow RMI violations (e.g., the "interactive" race model of Mordkoff \& Yantis, 1991). For these models, it is said that the RMI is violated because the racers run at different speeds in single and redundant trials-violating an assumption of the RMI known as "context independence" (e.g., Luce, 1986) or "context invariance" (e.g., Gondan \& Minakata, 2015) - rather than because there is coactivation. ${ }^{1}$ This has led to confusion about (a) which race models do and do not require the RMI to be satisfied, (b) what models are or are not compatible with observed RMI violations, and (c) how RMI

\footnotetext{
${ }^{1}$ As explained by Gondan and Minakata (2015), the assumption that racers run at the same speed in all conditions is different from the assumption that the racers' finishing times are stochastically independent (i.e., uncorrelated). The assumption of stochastic independence is of little concern because it is not required for the RMI. Since the assumption of context independence is required, redundancy gain studies are typically designed to minimize the opportunities for racers to run at different speeds in different stimulus conditions (e.g., by using mixed rather than blocked stimulus presentations; Gondan \& Minakata, 2015).
} 
violations should be interpreted with respect to models with separate activation versus coactivation.

In this note, I try to clear up some of this confusion by examining the space of possible models for the RSE. In particular, I focus on the assumption of context independence and the relationship between violations of this assumption and coactivation. In brief, I argue that context dependence is actually a type of coactivation, not an alternative to it.

\section{Race models and statistical facilitation}

As introduced by Raab (1962), the essential idea of race models is that the RSE arises due to statistical facilitation. To make this idea concrete, consider two hypothetical $10 \mathrm{~K}$ runners, who may be denoted as " $\mathrm{A}$ " and " $\mathrm{V}$ " to emphasize the connection between the race metaphor and the redundant signals paradigm with auditory and visual signals. Suppose that A and V each run the $10 \mathrm{~K}$ course on numerous occasions. On some occasions, they run alone, or "solo" (analogous to single-signal trials), whereas on other occasions they run simultaneously, or in "races" (analogous to redundant-signal trials). Assume further that the solo runs and races are randomly intermixed, like trials in a redundant-signals experiment, so that $\mathrm{A}$ and $\mathrm{V}$ cannot prepare differently for these two types of runs.

Now suppose that a sports researcher compares the average finishing time for each runner's solo runs with the average finishing time of the winners of the races, which is analogous to the RT researcher's comparison of the mean single- and redundant-signal RTs. The sports researcher might find that the winners' times were faster, on average, than the mean solo time for either racer, so that there was a "winner's advantage" analogous to the RSE. How might this advantage be explained?

One fairly simple explanation says that the advantage is "purely statistical" in the sense discussed by Raab (1962). According to this explanation, the winning time of each race is the minimum of two separate random variables - that is, the running times of the two racers $\mathrm{A}$ and $\mathrm{V}$, respectively. If the distributions of these running times overlap, then it can be shown that the average winner's time will-for purely statistical reasons - be less than the mean of either solo distribution (e.g., Miller \& Ulrich, 2003, footnote 8). This is "statistical facilitation." In a sense, Raab's race model represents a kind of baseline winner's advantage (RSE) that emerges even if "nothing special" happens in the races. ${ }^{2}$ Note, too, that this

\footnotetext{
${ }^{2}$ Raab (1962) formally considered only models in which the runners' finishing times were stochastically independent, so Miller (1982) introduced the term separate-activation models for the broader category of possibly- dependent race models. Because Raab's description of the race framework generalizes so nicely to models with correlated finishing times, however, the new terminology was largely ignored, and future researchers have usually used race models to include those allowing stochastic dependence.
}

statistical facilitation account of the winner's advantage implicitly assumes that the finishing time distributions of $\mathrm{A}$ and $\mathrm{V}$ are the same in the races as in the solo runs. That is, it says that the average winner's time is less only because there is some advantage for the faster of two times when the distributions overlap. Thus, Raab's model of statistical facilitation already includes the assumption of context independence that is required in order to derive the RMI.

The RMI provides one way to test this statistical facilitation account of the winner's advantage. As is described in detail by Gondan and Minakata (2015), it uses the solo runs to estimate the full probability distributions of finishing times for the two runners $\mathrm{A}$ and $\mathrm{V}$. Those two distributions can then be used to compute an upper bound distribution for the cumulative probability distribution of the winner's finishing times. ${ }^{3}$ If the winner's advantage (RSE) is just due to the statistical facilitation considered by Raab (1962), then the observed cumulative probability distribution of the winners' finishing times must be less than or equal to the upper bound distribution provided by the RMI, at every time point for which the observed and bound distributions are compared.

On the other hand, if the RMI is not satisfied (i.e., if there are RMI violations), then statistical facilitation cannot be the full explanation of the winner's advantage. In this case, something special must be happening in the races to produce faster times than one would have expected from the solo runs. Some other, probably more complex, explanation must be found for the winner's advantage. One possibility, for example, is that A's and V's competitive spirits motivate them to run faster in races than they do in their solo runs. If that happens, then their individual race times would be especially fast, and the winner's advantage would not be purely statistical. A more elaborate model, incorporating motivational effects, as well as statistical facilitation, would then be needed to account for the winner's advantage.

With respect to the aims of this paper, the key question is whether it is still appropriate to use the term "race model" to describe this more elaborate model including motivational effects. It is very tempting to do so; the model does, after all, describe the outcomes of races. More specifically, one might say that this is still a race model, but the assumption of context independence is violated by virtue of the fact that $\mathrm{A}$ and $\mathrm{V}$ run

\footnotetext{
${ }^{3}$ As is discussed in more detail by Gondan and Minakata (2015), an important technical feature of the RMI is that it applies whether the racers' finishing times are stochastically dependent (i.e., correlated) or not. Only a bound can be computed - not an exact prediction - because the degree of correlation between the two runners' finishing times does have an effect. For example, there might be a positive correlation if both runners respond the same way to daily temperature fluctuations, and this would produce a relatively small winner's advantage. Alternatively, there might be a negative correlation if one runner does better when it is cold and the other does better when it is hot, and this would produce a relatively large winner's advantage.
} 
faster when racing against each other than when running solo. Conveniently, in the normal race scenario, one could objectively document the runners' speed-up in races, since each runner's individual finishing times would be observable in the races as well as in the solo runs.

Despite the temptation to call this a "race model," I think that theoretical confusion arises from doing so. It should get a different name-perhaps "race plus motivation"-because it does not attribute all of the winner's advantage to statistical facilitation as Raab's (1962) race model would. Furthermore, it does not have to satisfy the RMI. Calling this a race model leads to confusion because it suggests that there are two different reasons why the RMI might fail: (1) coactivation, and (2) context dependence. Instead, I believe that context dependence should be considered a type of coactivation, not an alternative to it. Gondan and Minakata (2015) also view context dependent models as a "second class of coactivation models ... [that] drops the notion of context invariance (Equation 4)".

Consider the example with $10 \mathrm{~K}$ runners in more detail. What is the functional implication of the fact that $\mathrm{A}$ and $\mathrm{V}$ are faster when racing than when running solo (i.e., of the fact that there is context dependence)? It necessarily means that something about the presence of $\mathrm{V}$ causes $\mathrm{A}$ to speed up, and vice versa (e.g., due to motivational influences). In other words, both runners have an influence on the finishing time in a single race - one directly, by winning, and the other indirectly, by making the winner run faster than normal. This means that the outcome of the race is not determined solely by a single runner, and it represents a type of "coactivation" in the sense that "both components [runners] of a redundant signal [race] . . . influence responding on a single trial" (Miller, 1982, p. 248). In the terms often used in RT modeling, the context dependence could be said to imply that V's presence somehow provides extra "activation" to $\mathrm{A}$, thus allowing $\mathrm{V}$ to have an influence on A's winning time.

In summary, this example illustrates two points: (a) A situation involving runners and a physical race need not satisfy Raab's (1962) criteria for a "race model" producing purely statistical facilitation. For example, a race is not adequately described by a Raab-type race model if the performance of the runners changes when there is competition. (b) Context dependence implies that the presence of one racer influences the finishing times of the other racer. As a result of these between-racer influences, both racers influence the winning time for each race, which is essentially the definition of coactivation. Thus, RMI violations always justify the conclusion that both racers contributed to the race's outcome, even if these violations arose because of context dependence.

\section{Psychology and neurophysiology}

The arguments of the preceding section were framed in terms of a hypothetical race involving $10 \mathrm{~K}$ runners, so it seems important to ask how well the arguments would generalize to the sorts of psychological and neurophysiological detection and response processes actually involved in typical redundantsignals experiments. If anything, the arguments seem even stronger in these cases.

As a prototypical example, consider a simple audio-visual detection task of the sort discussed by Gondan and Minakata (2015). Suppose that the RMI is violated, ruling out pure statistical facilitation. Researchers in group A conclude that the two senses coactivate the responses in redundant trialsthat is, both stimuli influence responding in a single redundant trial. Researchers in Group B dispute that conclusion, however, claiming that the RMI violations arose because of context dependence.

What exactly is the issue in this dispute? Imagine that researchers in Group B developed a sophisticated neural monitoring apparatus that allowed them to show (a) the visual and auditory signals really do activate responses through two distinguishable vision-toresponse and audition-to-response channels, (b) that the RT in a redundant trial is determined by the first of these two channels to finish, and (c) the RMI is violated because of context dependence; that is, the detection on each channel is faster when the other modality's signal is present than when it is absent. This would be impressive evidence for context dependence, and it would greatly improve our understanding of the RSE observed in this case. Furthermore, these findings would tell researchers that they had to characterize the crosschannel interactions before they could fully understand the system. They might try to do that, for example, by looking for pathways supporting the interchange of activation between the two channels.

The findings would not, however, be evidence against coactivation. Coactivation models are defined by the idea that both stimuli can influence the response on a single trial. If the speed of detecting the visual signal depends on whether the auditory signal is present, then the auditory signal must have an influence, in one way or another, on the speed of visual detection, and vice versa. Theoretically, these between-channel influences are often modeled as each channel providing "activation" to the other channel, so the term "coactivation" seems a convenient short-hand for models that allow such influences. As in the case of the $10 \mathrm{~K}$ runners, though, even the hypothesized evidence that the RMI violations arose from context dependence would only further specify how coactivation took place, not contradict coactivation or provide an alternative account. 


\section{Context dependent race models are not falsifiable}

As a counter to the arguments of the previous sections, one might argue that it is important to maintain a separate category for what might be called "context dependent race models" because these models provide a class of explanations for the RSE that is more restricted than the open-ended class of coactivation models. The purpose of this section is to show that this is an illusion. Actually, the class of context dependent race models is sufficiently open-ended that it can never be falsified, just like the wider class of coactivation models. Thus, context dependent race models are not fundamentally more falsifiable than coactivation models.

Formally, context dependent race models can be characterized by five random variables: $A_{s}, V_{s}, A_{r}, V_{r}$, and $W_{r}$. The variables $A_{s}$ and $V_{s}$ represent the finishing times of the racers in the single-signal conditions, $A_{r}$ and $V_{r}$ represent the finishing times of the racers in the redundant condition, and $W_{r}$ represents the finishing times of the winner of the race. The idea of a race implies that

$W_{r}=\min \left\{A_{r}, V_{r}\right\}$.

If context independence is not assumed and $A_{r}$ and $V_{r}$ are free to have any distributions, then Equation 1 can always be satisfied. For a simple existence proof of this claim, suppose that $A_{r}$ and $V_{r}$ are stochastically independent, identically distributed random variables with $F_{A_{r}}(t)=F_{V_{r}}(t)=1-\sqrt{1-F_{W_{r}}(t)}$, where $F_{A r}(t), F_{V r}(t)$, and $F_{W r}(t)$ are the cumulative distribution functions of the random variables $A_{r}, V_{r}$, and $W_{r}$, respectively. Then,

$$
\begin{aligned}
F_{W_{r}}(t) & =\mathrm{P}\left[\min \left\{A_{r}, V_{r}\right\}<t\right] \\
& =\mathrm{P}\left[A_{r}<t\right]+\mathrm{P}\left[V_{r}<t\right]-\mathrm{P}\left[A_{r}<t\right] \times \mathrm{P}\left[V_{r}<t\right] \\
& =2 \times\left[1-\sqrt{1-F_{W_{r}}(t)}\right]-\left[1-\sqrt{1-F_{W_{r}}(t)}\right]^{2} \\
& =F_{W_{r}}(t) .
\end{aligned}
$$

This shows that absolutely any observed distribution of $W_{r}$ can be explained perfectly within a context dependent race model by setting both single-signal distributions to $1-\sqrt{1-F_{W_{r}}(t)}$, and many other assumptions about the single-signal distributions will also work. The point is that one can always find a model satisfying Equation 1 to fit any observed distribution of redundant RTs; this class of models can never be ruled out. The assumption of context independence (i.e., $A_{s} \equiv A_{r}$ and $V_{s} \equiv V_{r}$ ) is one plausible constraint that can be added to Equation 1 to produce falsifiable models, and this constraint leads to the RMI. Other constraints could also be considered, but some additional constraints are essential if context dependent race models are to be falsifiable.

\section{Conclusions}

The proper interpretation of RMI violations depends on an appropriate characterization of the models that do and do not require it to be satisfied. The former are often called "race models" following Raab (1962), but they might better be called "context-independent race models" to emphasize the assumption that the racers have the same distributions of finishing times in the single and redundant conditions. The latter are often called "coactivation" models, and this broad class was defined as including any model in which a single redundant response is - in one way or another - influenced by both channels (Miller, 1982). The coactivation class includes "context dependent race models," in which each individual racer's speed is influenced by the stimuli on both channels. Context dependent race models can always fit perfectly the RT distribution observed in a redundant-signal condition, so they are not falsifiable without further constraints.

Author Note I am grateful to the Alexander von Humboldt foundation for support during the preparation of this manuscript and to Matthias Gondan, Patricia Haden, Wolf Schwarz, and an anonymous reviewer for constructive comments on earlier versions of the article.

\section{References}

Gondan, M., \& Minakata, K. (2015). A tutorial on testing the race model inequality. Attention, Perception, \& Psychophysics. doi:10.3758/ s13414-015-1018-y

Luce, R. D. (1986). Response times: Their role in inferring elementary mental organization. Oxford, England: Oxford University Press.

Miller, J. O. (1982). Divided attention: Evidence for coactivation with redundant signals. Cognitive Psychology, 14, 247-279. doi:10.1016/ 0010-0285(82)90010-X

Miller, J. O., \& Ulrich, R. (2003). Simple reaction time and statistical facilitation: A parallel grains model. Cognitive Psychology, 46, 101151. doi:10.1016/S0010-0285(02)00517-0

Mordkoff, J. T., \& Yantis, S. (1991). An interactive race model of divided attention. Journal of Experimental Psychology: Human Perception \& Performance, 17, 520-538. doi:10.1037//0096-1523.17.2.520

Raab, D. H. (1962). Statistical facilitation of simple reaction times. Transactions of the New York Academy of Sciences, 24, 574-590.

Schwarz, W. (1989). A new model to explain the redundant-signals effect. Perception \& Psychophysics, 46(5), 498-500. doi:10.3758/ BF03210867

Schwarz, W. (1994). Diffusion, superposition, and the redundant-targets effect. Journal of Mathematical Psychology, 38, 504-520. doi:10. 1006/jmps. 1994.1036 